\title{
Journal of Membrane Biology: Cell Biology Section
}

\author{
Diane Lidke ${ }^{1}$
}

Published online: 18 April 2016

(C) Springer Science+Business Media New York 2016

The Journal of Membrane Biology has historically published studies on the role of membranes in regulating cell biology processes. This has included topics such as membrane protein structure-function, membrane-mediated signaling mechanisms, and cell-cell communication. The new section "Cell Biology" will continue these themes, while at the same time opening up to a broader range of topics. In this context, we are interested in manuscripts that focus on the crucial role for lipid membranes in all types of cellular processes, ranging from plasma membrane organization to vesicle trafficking to mechanical sensing.

It is now well recognized that the plasma membrane has a nanoscale organization. In the more than 40 years since Singer \& Nicholson put forth the Fluid Mosaic Model, a number of mechanisms through which the membrane is organized have been reported. This journal still aims at providing a platform for studies about lipid rafts, actin corrals, tetraspanins, and other nanoclustering mechanisms. Particularly, exciting is the potential role of the glycan moieties conjugated to proteins and lipids in regulating membrane organization and dynamics. An ultimate goal of this section is to increase our understanding of how membrane compartments regulate signal transduction in time and space.

Membrane organization within internal cellular compartments is also gaining importance. For example, nanodomains have been found in endosomes, putting forward the concept that endosomes are in fact intracellular signal transduction stations. Recent studies have demonstrated the existence of lipid domains in the nuclear membrane. In addition, specialized intracellular membrane organelle contact sites are an area of intense investigation, which we believe can find in our journal a new forum.

Common to all biomembranes are the still poorly understood topics of transbilayer coupling, leaflet asymmetry, and membrane curvature. While long-chain lipids have been implicated in the transbilayer coupling of the plasma membrane, the details of this process still remain to be elucidated. Moreover, it is unknown if similar coupling mechanisms exist in organelles. Membrane geometry is known to play an important role in membrane protein distribution, and specialized membrane invaginations such as caveolae have been implicated in sensing of mechanical cues. A current challenge is to increase our understanding of these physicalchemical principles and how they affect cellular outcomes.

Finally, we believe that quantitative studies integrating information from multiple disciplines are fundamental to advancing the field of membrane biology. Techniques such as lipidomics, organelle isolation, and high-resolution imaging have provided insights into the composition and organization of biomembranes with unprecedented detail. Therefore, the application of cutting-edge, quantitative techniques will have a prominent place in the pages of JMBi. We hope that our readership will identify with this newly defined journal section and help us to establish a vibrant new community in this field.
Diane Lidke

dlidke@salud.unm.edu

1 Department of Pathology, Cancer Research Facility, School of Medicine, University of New Mexico, Room 203, Albuquerque, NM 87131, USA 